\title{
Signal Separation and Synthesis in the Wigner-Ville Distribution Domain with Fractional Fourier Transform
}

\author{
Haitao Cao ${ }^{1, a}$, Gaoyong Luo ${ }^{1, b}$ \\ ${ }^{1}$ School of Physics and Electronic Engineering, Guangzhou University, Guangdong 510006, China \\ afightingcat@163.com, bgyl@gzhu.edu.cn
}

\begin{abstract}
Keywords Gaussian White Noise, Wavelet Shrinkage Denoising, Fractional Fourier Transform, Wigner-Ville Distribution, Signal Synthesis.
\end{abstract}

\begin{abstract}
In this paper we propose a new method to separate the composite signal which consist of sinusoidal signals and chirp signal utilizing the time-frequency (TF) window in the Wigner-Ville Distribution (WVD) domain. The signal is reconstructed from the TF domain based on the Wigner Distribution Synthesis (WDS) techniques. An impulse is obtained from linear chirp signal after using Fractional Fourier Transform (FrFT) with an optimal rotate angle in the TF domain. The impulse is windowed to extract chirp signal. Wavelet Shrinkage Denosing (WSD) technique is developed as a preprocessing of original signal to suppress the Gaussian white noise. Simulation results show that the proposed method performs better in terms of separation of sinusoidal signals and chirp signal which overlap in both time and spectra.
\end{abstract}

\section{Introduction}

Traditional noisy signal separation can be considered as a filtering process, where time domain signal is often transformed to frequency domain for further analysis and processing. However, in real world, the signals in time domain and frequency domain are arbitrary overlapped, so that it is difficult to extract useful features and effectively separate real signal and noise by the traditional filter design method. The basic principle of time-frequency (TF) analysis is to design the joint function of time and frequency, and to describe the energy density and strength of signal in both time and frequency domain. One of the mostly used TF representations is Wavelet Transform (WT). Donoho proposed the Wavelet Shrinkage Denosing(WSD) in 1995 [1], since then a lot of signal detection and separation work has been done with additive Gaussian white noise as background noise. Research has shown that Wavelet Shrinkage Denosing (WSD) is a good choice for filtering Gaussian white noise with less signal distortions. The Wigner Distribution (WD) has also been used as a mixed time-frequency signal analysis tool.

For chirp signal, because of its high value wavelet coefficients and strong TF coupling with other signals, the WSD and the classical filtering method are both difficult to effectively achieve the chirps separation. The definition of Fractional Fourier Transform (FrFT) was firstly proposed from the perspective of mathematical by Namias V in 1980 [2]. The interpretation of rotation operator on the $\mathrm{TF}$ plane was presented by Almeida L B, analyzing the relationship between FrFT and Wigner-Ville Distribution (WVD) [3]. Research has demonstrated that the FrFT can be used as a rotary tool of TF plane and that the transformation is especially suitable for processing chirp signal. The separation of linear chirps with the FrFT is not new and a lot of work has been done and presented [4-6]. Based on short-time FrFT, a pseudo WVD is proposed to analyze and separate multi-component chirp signals [4]. However, the algorithm has excessive computation to obtain the optimal rotate angle and the reconstruction in time domain cannot be achieved. The multi-component chirp signals are also separated in [5] but its signal phase is distorted. The separation and denoising of chirp signals have also been implemented with FrFT based on the WVD but the signal cannot be reconstructed in time domain [6].

This paper proposes a new method for signal separation and synthesis. We formulate the 
method to obtain the optimal rotate angle of chirp in the fractional domain. With the application of FrFT based on the WVD, the signal separation is achieved more effectively.

\section{Preliminaries}

Nonlinear Wavelet Threshold Denoising Method.In wavelet domain, the values of wavelet coefficients corresponding to the signal are higher. As a result, a threshold can be set to distinguish signal and noise wavelet coefficients. The values of wavelet coefficients of noise tend to be lower than the threshold and they can be set to zeros for noise reduction. The remainder coefficients are regarded as the wavelet coefficients of the signal and can be used to reconstruct back the denoised signal. This method is computationally efficient and easy to implement.

Wigner Distribution Synthesis Techniques.Discrete-time Wigner Distribution (DTWD) is proposed by Claasen [7]:

$$
\hat{W}_{x}(n, \omega)=2 \mathrm{~T} \sum_{m=-\infty}^{\infty} \mathrm{x}(n+m) \mathrm{x}^{*}(n-m) e^{-j 2 \omega m T},|\omega|<\frac{\pi}{2 \mathrm{~T}}
$$

where $\mathrm{T}$ is the sampling period. Equation (1) may be rewritten as

$$
\begin{aligned}
\hat{W}_{x}(n, \omega) & =2 T \sum_{r=-\infty}^{\infty} \mathrm{x}(2 r) \mathrm{x}^{*}(2(n-r)) e^{-j 2 \omega(2 \mathrm{r}-n) T} \\
& +2 T \sum_{r=-\infty}^{\infty} \mathrm{x}(2 r-1) \mathrm{x}^{*}(2(n-r)+1) e^{-j 2 \omega(2 \mathrm{r}-n-1) T},|\omega|<\frac{\pi}{2 \mathrm{~T}}
\end{aligned}
$$

The first summation depends on only the even-indexed samples and the second depends only on the odd-indexed samples. The Wigner synthesis problem can be defined as follows: given a target WD, find a signal whose WD is closest to the target distribution.

Find the digital sequence $\mathrm{x}(n)$ whose DTWD, $\hat{W}_{x}(n, \omega)$, best approximates, in a least squares sense, given WD $Y(n, \omega)$. In other words, find the finite-energy signal $\mathrm{x}(n)$ that minimizes [8]

$$
J(x)=\sum_{n} \frac{\mathrm{T}}{\pi} \int_{-\pi / 2 T}^{\pi / 2 T}\left|Y(n, \omega)-\hat{W}_{x}(n, \omega)\right|^{2} d \omega
$$

Using Parseval's theorem it can be rewritten as

$$
\begin{aligned}
J(x)= & \sum_{n} \sum_{m}\left|\mathrm{y}(n, m)-2 T \mathrm{x}(n+m) \mathrm{x}^{*}(n-m)\right|^{2} \\
= & {\left[\sum_{n} \sum_{r}\left|\mathrm{y}(n, 2 r-n)-2 T \mathrm{x}(2 r) \mathrm{x}^{*}(2 n-2 r)\right|^{2}\right] } \\
& +\left[\sum_{n} \sum_{r}\left|\mathrm{y}(n, 2 r-1-n)-2 T \mathrm{x}(2 r-1) \mathrm{x}^{*}(2 n-2 r+1)\right|^{2}\right]
\end{aligned}
$$

where $\mathrm{y}(n, m)$ is the inverse Fourier transform of $Y(n, \omega)$.

The optimal minimizer of (4) can be derived in a three-step procedure. In step 1, the even-indexed samples of the optimal signal $\mathrm{x}(n)$ are found. Similarly, in step 2, the odd-indexed samples of the optimal $\mathrm{x}(n)$ are derived. The third step derives the phase $\alpha$ of the estimated signal.

Fractional Fourier Transform. There is a direct relationship between FrFT and TF signal analysis. The WD of the FrFT result of $\mathrm{x}(t)$, is the WD of $\mathrm{x}(t)$, rotated by an angle $-\alpha$. If the signal has a chirp representation, the FrFT can be used to find the corresponding angle that would allow us to change the signal into a sparse representation of it. Correspondingly, the optimal transform order which changes the linear chirp signal to be an impulse in the fractional domain can be written as $[5,7,8]$

$$
p_{\text {opt }}=-\frac{2}{\pi} \tan ^{-1}\left(\frac{f_{s}}{B}\right)
$$


where $B$ is the bandwidth in hertz and $f_{S}$ is sampling frequency.

Phase Trimming.From the WDS it is noted that the phase information of synthesized signal should be estimated by the original signal. When we window the impulse after rotating the chirp signal in the TF domain, the windowed signal need to be transformed from TF domain to time domain. In this process, we can not refer to the original signal. So serious phase distortion can take place on synthesized signal after $\mathrm{iFrFT}$ in time domain when compared to original signal.

We propose a novel scheme to solve this problem. We make cross-correlation between synthesized signal and original signal, and obtain the maximum cross-correlation value. Signals superpose at the moment when this value appears. Then the delay of two signals is the error of the superposition moment and the number of sample points. At last we complete phase trimming by resetting the synthesized signal with the delay.

\section{Algorithm Implementation}

Signal separation and synthesis in the WVD domain using FrFT is a four-step process:

(1) Preprocess the noisy signal with WSD and suppress Gaussian white noise; (2) Window the WVD representation of the preprocessed signal with rectangular window. Reconstruct the sinusoidal signal in time domain with WDS; (3) Derive the optimal transform order for the remaining noisy chirp signal based on equation (5) Perform FrFT and window the impulse in the fractional domain with rectangular window; (4) The synthesized signal in time domain can be derived with WDS on the windowed impulse function in fractional domain. Then iFrFT the synthesized signal and the result is reconstructed with phase of chirp signal trimmed. Fig. 1 illustrates the proposed method with TF plane representations of the signals at each step of the process.

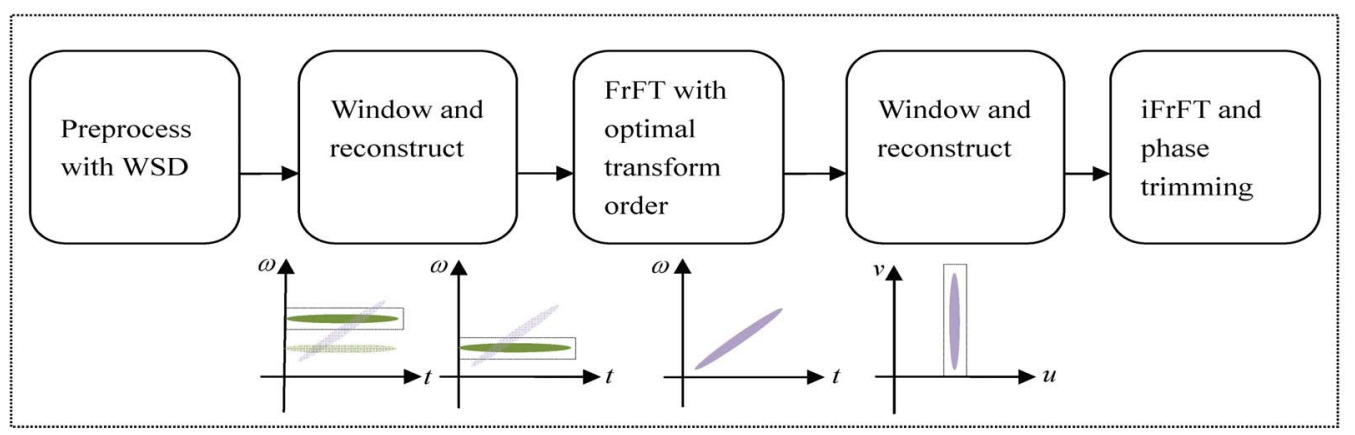

Fig. 1 Signal flow diagram illustrating the four steps required for signal separation and synthesis in the WVD domain using FrFT.

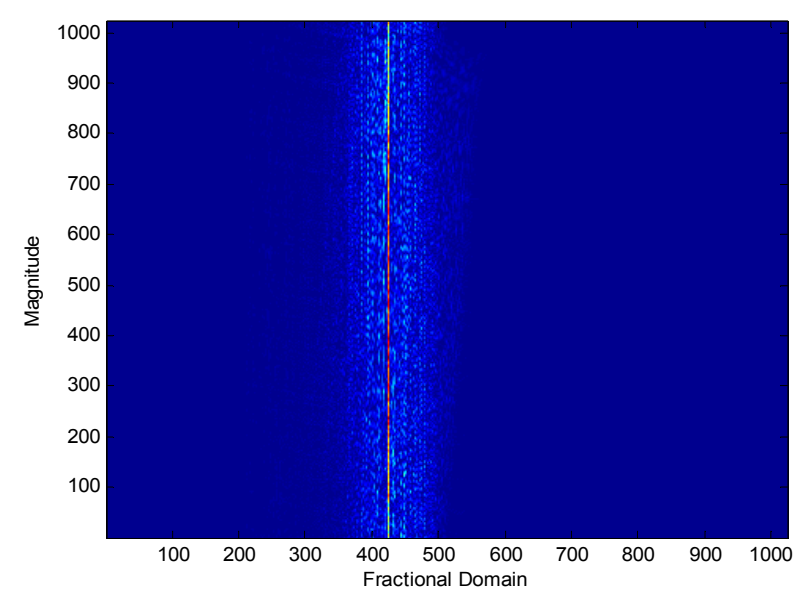

Fig. 2 The impulse function rotated from slash representation with the optimal rotate angle in fractional domain. 


\section{Simulation}

Consider a composite signal

$$
\mathrm{x}(t)=\mathrm{n}_{1}(t)+\mathrm{n}_{2}(t)+\mathrm{c}(t)+\mathrm{g}(t)
$$

where, $\mathrm{n}_{1}(t)$ and $\mathrm{n}_{2}(t)$ is sinusoidal signal with the frequency of $100 \mathrm{~Hz}$ and $200 \mathrm{~Hz}$, respectively. $\mathrm{c}(t)$ is linear chirp signal whose frequency ranges from $50 \mathrm{~Hz}$ to $300 \mathrm{~Hz}$. $\mathrm{g}(t)$ is Gaussian white noise. After the processing of the suggested four-step algorithm, the average values of correlation coefficients of the reconstructed signal and the original signal are shown in Table 1 . The optimal transform order of the noisy chirp signal is $p=-0.9208$ in fractional domain. An impulse appears in the fractional domain by rotating the optimal transform angle, as shown in Fig. 2.

Table 1 The average values of correlation coefficients of the reconstructed signal and the original signal in the two cases of no-reference and reference.

\begin{tabular}{|c|c|c|}
\hline correlation coefficients (average) & No-Reference & Reference \\
\hline Sinusoidal signal 1 & 0.1419 & 0.9740 \\
\hline Sinusoidal signal 2 & 0.4839 & 0.9813 \\
\hline Linear chirp signal & -0.0897 & 0.8641 \\
\hline
\end{tabular}

\section{Conclusions}

This paper presents an algorithm to separate the composite signal in the WVD domain using FrFT and implement synthesis with WDS techniques. The signal is first decomposed into wavelet domain and the wavelet coefficients are thresholded on different frequency levels. After reconstructing the processed signal, the Gaussian white noise can then be suppressed. Simulation results show the effectiveness of this algorithm in separating the different components of a signal in the Wigner time-frequency domain. Not only the composite signal can be separated, but also the noise and the cross terms are suppressed. The results reported demonstrate the potential of the proposed method to extract the features of signal.

\section{References}

[1] D L Donoho: Denosing by soft-thresholding. IEEE Transactions on Information Theory, 41(3):613-627,(1995).

[2] Namias V: The fractional Fourier transform and its application in quantum mechanics. IMAJ of Appl Math , 241-265, (1980).

[3] Almeida L B: The fractional Fourier transform and time-frequency representation. IEEE Trans on SP,42 (11 ):3084-3091,(1994).

[4] Y.L.Zang, F.Q.Yu: Multi-component Chirp signal separation using pseudo Wigner-Ville distribution in short-time fractional Fourier domain. Computer Engineering and Applications, 50 (4): 211-214,(2014).

[5] Cowel D M J,Freear S: Separation of overlapping linear frequency modulated (LFM) signals using the fractional fourier transform. IEEE Transactions on Ultrasonics, Ferroelectries, and Frequency Control,57(10):2324-2333, (2010).

[6] O.A.Alkishriwo, L.F.Chaparro,A.Akan: Signal separation in the Wigner distribution domain using fractional Fourier transform. Proc.19th Eur.Signal Process.Conf., Barcelona, Spain, Aug. 29/Sep.2, pp.1879-1883, (2001).

[7] T.A.C.M.Claasen, W.F.G.Mecklenbrauker: The aliasing problem in discrete-time Wigner distributions. IEEE Trans. Acoust., Speech. Signal Processing, vol. ASSP-31, pp. 1067-1072, Oct. (1983).

[8] G.F.Boudeaux-Bartels, T. W. Parks: Time-varying filtering and signal estimation using Wigner distribution synthesis techniques. IEEE Trans.ASSP,vol,34,pp.442-451,June (1986). 